\title{
Probiotics do not reduce antibiotic-associated or Clostridium difficile diarrhoea in older hospitalized patients
}

Antibiotic-associated diarrhoea (AAD) occurs frequently in older patients ( $\geq 65$ years), and can be life threatening. "Microbial preparations (often referred to as probiotics) have been previously assessed in prevention of AAD and the evidence from several smaller trials was quite encouraging," explains Professor Stephen Allen, a researcher at Swansea University, Wales.

\section{Thur findings should discourage the use of microbial preparations for the prevention of AAD... 77}

As reported in The Lancet, Allen and colleagues conducted a multicentre, randomized, placebo-controlled, pragmatic study to evaluate the efficacy of a microbial preparation (comprising two strains of lactobacilli and two strains of bifidobacteria) for prevention of AAD in hospital inpatients aged $\geq 65$ years who had been exposed to at least one oral or parenteral antibiotic. Patients were given either probiotic $(n=1,470)$ or placebo $(n=1,471)$ for 21 days and followed up for 8-12 weeks.

Overall, AAD occurred in $~ 10 \%$ patients, with diarrhoea caused by $C$. difficile in $\sim 1 \%$ of patients; these outcomes were equally common in those taking probiotic and placebo. Factors such as common gastrointestinal symptoms, length of hospital stay and quality of life were also similar in the two groups. "So, there was no evidence that the microbial preparation had prevented diarrhoea or had led to any other health benefit," Allen states.

Of note, no difference in rates of serious adverse events were reported between patients given probiotic and those given placebo ( $20 \%$ of patients in each group), indicating that the use of this microbial preparation in this setting is safe.
"Our findings should discourage the use of microbial preparations for the prevention of AAD and Clostridium difficile diarrhoea," says Allen.

Further research is required to determine the underlying mechanisms of AAD. "A major problem is that there is a potentially very large number of probiotics and probiotic combinations that could be evaluated in clinical trials. We would advise against clinical trials of other microbial preparations - unless there is some reliable evidence (such as from laboratory studies) that a specific preparation may directly address known underlying mechanisms," concludes Allen.

Katherine Smith

Original article Allen, S. J. et al. Lactobacilli and bifidobacteria in the prevention of antibiotic-associated diarrhoea and Clostridum difficile infection in older inpatients (PLACIDE): a randomised, double-blind, placebo-controlled, miulticentre trial. Lancet doi:10.1016/ S0140-6736(13)61218-0 